\title{
Facile synthesis of copper ions chelated sand via dopamine chemistry for recyclable and sustainable catalysis
}

\author{
Ning Wang a , Zhongren Zhang a , Juanru Huang ${ }^{\mathrm{b}}$, Yunxia $\mathrm{Hu}^{\mathrm{b}, *}$ \\ ${ }^{a}$ CAS Key Laboratory of Coastal Environmental Processes and Ecological Remediation, Yantai Institute of Coastal Zone Research, Chinese Academy of Sciences, Yantai, \\ Shandong Province 264003, PR China \\ ${ }^{\mathrm{b}}$ State Key Laboratory of Separation Membranes and Membrane Processes, National Center for International Research on Membrane Science and Technology, School of \\ Materials Science and Engineering, Tianjin Polytechnic University, Tianjin 300387, PR China
}

\section{H I G H L I G H T S}

- $\mathrm{Cu}$ ions chelated sand (Cu-PDA@Sand) was simply synthesized through dopamine chemistry.

- Low-cost Cu-PDA@Sand exhibited efficient catalytic reduction properties towards organic dyes in the presence of $\mathrm{NaBH}_{4}$.

-Cu-PDA@Sand could be recovered by self-sedimentation and reused for 20 cycles.

- Cu-PDA@Sand presented long-term high catalytic activities.

\section{A R T I C L E I N F O}

\section{Article history:}

Received 4 December 2018

Received in revised form 28 March 2019

Accepted 5 April 2019

Available online 5 April 2019

\section{Keywords:}

$\mathrm{Cu}$ ions

Dopamine

Catalytic reduction

Organic dyes

Color removal
G R A P H I C A L A B S T R A C T

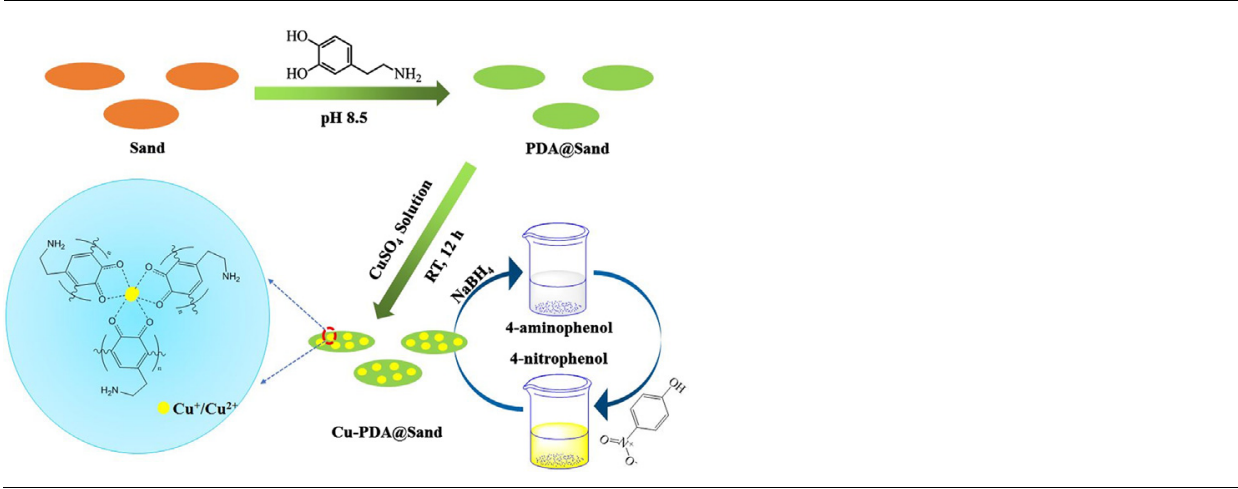

\section{A B S T R A C T}

Metal-based catalysts present highly efficient catalytic activities towards organic dyes and their intermediates for color removal. However, several issues are needed to be addressed urgently for their large-scale practical applications, such as the cost, stability and recyclable use of these catalysts. In this work, we developed a facile approach to chelate $\mathrm{Cu}$ ions on the polydopamine-coated sand (Cu-PDA@Sand) and investigated their catalytic properties towards the decoloration of organic dyes or intermediate including 4-nitrophenol (4-NP), methylene blue (MB), and congo red (CR). Our results found that the copper ions from Cu-PDA@Sand were the mixture of $\mathrm{Cu}$ (I) and $\mathrm{Cu}$ (II), and the molar fraction of $\mathrm{Cu}$ (I) over $\mathrm{Cu}$ (II) was increased from the original 3:2 to 4:1 in the presence of $\mathrm{NaBH}_{4}$. The reduction of these organic dyes or intermediate followed the first order kinetics, and the apparent reduction rate $k_{a p p}$ of CR was larger than that of MB and 4-NP under similar conditions. Additionally, the optimized $k_{a p p}$ of 4-NP could reach to $1.28 \mathrm{~min}^{-1}$, which is much higher than the reported results from literature. The recyclability test of $\mathrm{Cu}-$ PDA@Sand showed that the reduction efficiency of Cu-PDA@Sand maintained 100\% during the successive 20 cycles. Additionally, Cu-PDA@Sand was demonstrated to present long-term stability and catalytic activities after soaking Cu-PDA@Sand in water for 30 days. Our research demonstrated that the easyprepared low-cost Cu-PDA@Sand catalyst would have a great potential in the practical applications for color removal of the dye-containing wastewater.

두 2019 Elsevier Ltd. All rights reserved.

\footnotetext{
* Corresponding author.

E-mail address: yunxiahu@yic.ac.cn (Y. Hu).
} 


\section{Introduction}

Organic dyes are widely used not only in the textile industry but also in papers, plastics, rubbers, drugs, foods, paints, printings, and cosmetics (Ali, 2012; Okesola and Smith, 2016). However, tons of colored wastewaters containing organic dyes were produced every year and thus caused a serious aquatic ecosystem pollution, particularly, most of the organic dyes are carcinogenic, bright-colored, and un-degradable (de Luna et al., 2014; Eggen et al., 2014; Subair et al., 2016). In the past few years, different strategies have been developed to treat organic dyes in aqueous environment, including adsorption (Ayati et al., 2016; Hassan and Carr, 2018; Lipatova et al., 2018; Wang et al., 2018; Zhang et al., 2019), membrane separation (Kurt et al., 2012; Ong et al., 2014), chemical coagulation (Verma et al., 2012), biological methods (Laszlo, 2000), electrochemical processes (Szpyrkowicz et al., 2001), photochemical oxidation process (Sun et al., 2014), and catalytic reduction (Bastús et al., 2014; Panáček et al., 2014; Yao et al., 2014). The catalytic reduction of organic dyes using the metallic catalysts is considered as one of the most effective ways to remove color due to its fast and efficient performances. A variety of noble metals such as Au (Li et al., 2012; Yao et al., 2014), Ag (Zheng and Wang, 2012; Gao et al., 2016), and Pd (Chaplin et al., 2012) exhibit high catalytic activities of removing the color of organic dyes in the presence of reducing agents, because the noble metals can shift the Fermi level of electron donor to more negative potentials and thereby enhance the interfacial electron transfer to reduce organic dyes (Subramanian et al., 2004). Due to the expensive cost of noble metals, however, it is highly desired to develop cheap metallic catalysts with high activity and good stability.

$\mathrm{Cu}$-based catalysts have received increasing interests due to their outstanding catalytic properties and much low cost compared with noble metallic catalysts (Sun et al., 2013; Zhang et al., 2013; Zhang et al., 2014; Wang et al., 2017). Cu (0) nanoparticles exhibited a $0.39 \mathrm{~min}^{-1}$ of the high apparent rate constant $\left(k_{a p p}\right)$ towards 4-nitrophenol (Sun et al., 2013), which is similar or even higher than that of Au (Zhang et al., 2011; Luo et al., 2014), and Ag nanoparticles (Dong et al., 2014; Tang et al., 2015), respectively. Meanwhile, $\mathrm{Cu}_{\mathrm{x}} \mathrm{O}$ nanoparticles or clusters such as $\mathrm{Cu}$ (I) and $\mathrm{Cu}$ (II) oxides also have been demonstrated to promote the electron transfer from the donor to the acceptor, and then decolorate organic dyes efficiently in the presence of sodium borohydride (Sun et al., 2013). Unfortunately, nanoparticles, because of their high surface energy, easily clumped to form aggregation and difficultly recycled to use in the practical applications, and the agglomerate often destroyed their characteristic nanostructured properties and then severely deteriorated their catalytic activities (Xie et al., 2014).

Most recent investigations have been focused on the fabrication of substrate-supported catalysts to distribute the metal nanoparticles on the support for the prevention of their aggregation and the realization of their recyclable use. A variety of supports, including boron nitride (h-BN) (Huang et al., 2014), $\mathrm{Fe}_{3} \mathrm{O}_{4}$ (Feng et al., 2013), $\mathrm{Al}_{2} \mathrm{O}_{3}$ (Bradu et al., 2010), chitosan (Šuláková et al., 2007), mesoporous silica (SBA-15) (Ghosh et al., 2015), and graphene oxide (GO) (Hu et al., 2013) have been used as support materials to load copper nanoparticles. Among these various substrates, magnetic nanoparticles such as $\mathrm{Fe}_{3} \mathrm{O}_{4}$ could help to recover nanomaterials from wastewater in the presence of an external magnetic field (Feng et al., 2013; Zhu et al., 2013; Xie et al., 2014; Yao et al., 2014). Regretfully, the added extra separation and recovery process was not well engaged in the practical applications in industry or required high cost. Meanwhile, the fabrication procedure of these substrate supported catalysts often involves complicated processes, enduring time, rigorous conditions such as strong acid and base, organic solvents, high temperature, and so on. In addition, these supports were very expensive to be used for treating wastewater containing organic dyes. Therefore, it is urgently needed to select a support with low-cost and easy recovered properties and to develop a facile method of fabricating the $\mathrm{Cu}$-based catalyst on this support for the catalytic reduction of organic dyes.

In this work, we fabricated a cheap sand supported copper catalyst (Cu-PDA@Sand) and investigated the catalytic performances of $\mathrm{Cu}$ ions to remove the color of dye-containing water. Sand was selected as a support to chelate $\mathrm{Cu}$ ions because of its cheap cost and easy sedimentary properties from the aqueous environment. $\mathrm{Cu}$ ions were chosen as the metal catalyst due to its outstanding catalytic performance, very low cost, and non-aggregation properties in aqueous solution, compared with other noble metallic catalysts. The long-term catalytic activity and recyclability of $\mathrm{Cu}-$ PDA@Sand were estimated to develop its practical application possibility for the color removal of dyes in the water system.

\section{Experimental section}

\subsection{Chemicals}

Dopamine hydrochloride, Cupric sulfate $\left(\mathrm{CuSO}_{4}\right), p$-nitrophenol (4-NP, $\mathrm{C}_{6} \mathrm{H}_{5} \mathrm{NO}_{3}$ ), methylene blue ( $\mathrm{MB}, \mathrm{C}_{16} \mathrm{H}_{18} \mathrm{ClNS}$ ), Congo red (CR, $\left.\mathrm{C}_{32} \mathrm{H}_{22} \mathrm{~N}_{6} \mathrm{Na}_{2} \mathrm{O}_{6} \mathrm{~S}_{2}\right)$, and sodium borohydride $\left(\mathrm{NaBH}_{4}\right)$ were purchased from Sinopharm Chemical Reagent Co. Ltd. and used as received. Sand (collected from Yantai coast, China) was thoroughly washed with deionized water and filtered through 50 mesh size to remove large aggregates after $24 \mathrm{~h}$ dry in a vacuum oven at $80^{\circ} \mathrm{C}$.

\subsection{Preparation of polydopamine-coated sand (PDA@Sand) and $\mathrm{Cu}$ ions-chelated PDA@Sand (Cu-PDA@Sand)}

$100.0 \mathrm{~g}$ of sand was added in $2 \mathrm{~L}$ of DI water under magnetic stirring. After $30 \mathrm{~min}, 1.0 \mathrm{~g}$ of dopamine hydrochloride were added and $10 \mathrm{mM}$ Tris- $\mathrm{HCl}$ buffer was titrated to tailor the $\mathrm{pH}$ value of the above mixture at 8.5 . With the continuous stirring of $8 \mathrm{~h}$ at room temperature, the color of the sand suspension was turned from brown to dark with the polymerization of dopamine, and after 10 min sedimentation, the darken sand were collected followed by at least five times thorough washing with $25 \%$ isopropyl alcohol. Afterward, the dark solid dried at $80^{\circ} \mathrm{C}$ for $12 \mathrm{~h}$. Then $50.0 \mathrm{~g}$ of asprepared PDA@Sand was dispersed in $0.5 \mathrm{~L} \mathrm{CuSO}_{4}$ aqueous solution $(50 \mathrm{mM})$ under $12 \mathrm{~h}$ of stirring. Finally, the samples filtrated was repeatedly rinsed using DI water and dried at $60^{\circ} \mathrm{C}$ for at least $24 \mathrm{~h}$.

\subsection{Characterizations}

Samples were characterized with Fourier transform infrared spectrometer (FT-IR, Perkin-Elmer 2000, USA), Surface Area and Pore Analyzer (Nova 2200e, USA), scanning electron microscopy (SEM, S-4800, HITACHI, Japan) coupled with Energy-dispersive Xray (EDX), X-ray photoelectron spectroscopy (XPS, Thermo EscaLab 250Xi, Thermo Fisher Scientific, USA) (High-resolution scans were carried out for analysis of Cu element.), and UV-Vis spectrometer (PERSEE UT 1810, Shanghai, China).

\subsection{Catalytic reduction experiments}

The catalytic reduction capacity of the prepared Cu-PDA@Sand catalyst was fully examined by testing the catalytic reduction efficiency of organic dyes or intermediate, including 4-NP, MB, and CR. In general, 200 mg Cu-PDA@Sand, dye solution ( $1 \mathrm{~mL}$ of $1 \mathrm{~g} / \mathrm{L}$ ), and 
$\mathrm{NaBH}_{4}$ ( $2 \mathrm{~mL}$ of $0.1 \mathrm{M}$ ) aqueous solutions were completely mixed with $7 \mathrm{~mL}$ DI water under stirring. The time-dependent concentration of dye in the reacting solution was monitored by the UV-visible absorption spectra. The calibration curves of 4-NP, MB, and CR are shown in Fig. S1. Three key factors such as the concentrations of Cu-PDA@Sand, dye, and $\mathrm{NaBH}_{4}$ were investigated to study the reduction efficiency of dyes. Three key factors such as the concentrations of Cu-PDA@Sand, dye, and $\mathrm{NaBH}_{4}$ were investigated to study the reduction efficiency of dyes. The adsorption of the prepared Cu-PDA@Sand was investigated by monitoring the color removal of dyes in the absence of $\mathrm{NaBH}_{4}$, and the procedure is similar to the above catalytic reduction.

The apparent reduction rate constant $k_{a p p}$ of dyes was calculated through fitting a pseudo-first-order kinetic behavior based on the following Eq. (1) (Zeng et al., 2013):

$\ln \left(\frac{C_{t}}{C_{0}}\right)=-k_{\mathrm{app}} t$

where $k_{\text {app }}$ is the apparent reduction rate constant $\left(\min ^{-1}\right)$, and $\mathrm{t}$ is the equilibrium time. $C_{\mathrm{t}}$ and $C_{\mathrm{o}}$ are the concentration of the dyes at time $t$ and 0 , respectively.

The reduction efficiency $\left(\mathrm{E}_{\mathrm{dye}}\right)$ was calculated according to the Eq. (2):

$E_{\text {dye }}=\left(\frac{C_{0}-C_{t}}{C_{0}}\right) \times 100 \%$

where $\mathrm{E}_{\text {dye }}$ is the reduction efficiency of dye, and $C_{\mathrm{t}}$ and $C_{\mathrm{o}}$ are the concentration of the dyes at time $t$ and 0 , respectively.

\subsection{Long-term catalytic activity measurement}

After soaking in DI for 30 days, the catalytic activity of $\mathrm{Cu}^{-}$ PDA@Sand was examined. Typically, $1.0 \mathrm{~g}$ Cu-PDA@Sand and $40 \mathrm{~mL}$ of deionized water were mixed under shaking, and the asformed suspension was taken out and subsequently collected through self-sedimentation after 30 days. Upon the complete dry of Cu-PDA@Sand in a vacuum oven at $60^{\circ} \mathrm{C}$ for at least $24 \mathrm{~h}$, 200 mg Cu-PDA@Sand were mixed with dyes ( $1 \mathrm{~mL}$ of $1 \mathrm{~g} / \mathrm{L}$ ) (4nitrophenol, Congo red, and methylene blue, respectively), $\mathrm{NaBH}_{4}$ ( $2 \mathrm{~mL}$ of $0.1 \mathrm{M}$ ), and $7 \mathrm{~mL}$ DI water to measure its catalytic activity following the above-mentioned approach.

\subsection{Recyclability experiments}

The recyclability of Cu-PDA@Sand was explored to remove the color of 4-nitrophenol by successive 20 cycles. For the first cycle, 200 mg Cu-PDA@Sand, dye solution ( $1 \mathrm{~mL}$ of $1 \mathrm{~g} / \mathrm{L}$ ), and $\mathrm{NaBH}_{4}$ $(2 \mathrm{~mL}$ of $0.1 \mathrm{M})$ aqueous solutions were completely mixed with $7 \mathrm{~mL}$ DI water under stirring. The $k_{\text {app }}$ of dyes were successively measured and calculated based on the above-mentioned method. After the one cycle of reduction reaction was finished to completely remove the color of dyes, Cu-PDA@Sand was easily collected to regenerate through self-sedimentation, and then repeatedly utilized in the next cycle of reduction reaction, following the above conditions. The total recycles were extended to 20 times, and the $\mathrm{E}_{\mathrm{dye}}$ was carefully examined every five cycles.

\section{Results and discussion}

\subsection{Synthesis and characterization of Cu-PDA@Sand}

Dopamine chemistry provides a universal, facile, and green chemistry to coat polydopamine and thus chelate $\mathrm{Cu}$ ions on most of the support materials (Liu et al., 2014; Zhang et al., 2015; Zhang et al., 2017). Therefore, the fabrication scheme of Cu-PDA@Sand through dopamine chemistry is shown in Scheme 1. This procedure involved two steps: Firstly, the self-polymerization polydopamine occurred on the sand to obtain the PDA coated sand (PDA@Sand). Secondly, the as-prepared PDA@Sand chelated Cu ions through incubation with $\mathrm{CuSO}_{4}$ to synthesize Cu-PDA@Sand. In addition, upon the self-polymerization of dopamine on the sand (Scheme 1), the color of sand turned from pale yellow to dark as shown in Fig. 1a, which is a typical phenomenon to indicate that polydopamine was coated on the sand (Liu et al., 2016). In addition, the color of Cu-PDA@Sand did not change much upon 12 h immersion in the $\mathrm{CuSO}_{4}$ aqueous solution, which did not present a shiny color of metals upon the generation of metal nanoparticles on the PDA coated supports after incubation with inorganic salts (Liu et al., 2016; Wang et al., 2017).

FT-IR measurement was firstly performed to characterize the fabricated process. For the spectrum of PDA@Sand, the presentation of the strong peak at $1630 \mathrm{~cm}^{-1}$ was the typical adsorption peak of the amide groups, providing the evidence of the coating of PDA layer on sand (Fig. S2) (Wang et al., 2017). SEM coupled with EDX was employed to observe the surface morphologies of PDA@Sand and Cu-PDA@Sand, and to determine their surface elemental compositions, respectively. No obvious morphology change was found in their SEM images before and after PDA coating on sand (Fig. S3), which may due to a very thin layer of PDA coating formed on the sand. In addition, no particles were observed on the sand surface after incubating PDA@sand with the $\mathrm{CuSO}_{4}$ solution, which illustrates that PDA could not reduce $\mathrm{Cu}$ ions to generate $\mathrm{Cu}$ nanoparticles, agreeing with the reported results (Hu et al., 2013; Liu et al., 2016). As expected, nitrogen peak and $\mathrm{Cu}$ peak were detected upon PDA coating and $\mathrm{Cu}$ chelation based on their EDX spectra (Fig. S4). Furthermore, TGA results are shown in Fig. S4 to illustrate that sand is stable even under $800{ }^{\circ} \mathrm{C}$ with a weight loss of only $1.5 \mathrm{wt} \%$, and PDA can fully degrade at $800{ }^{\circ} \mathrm{C}$ with a weight loss of $100 \mathrm{wt} \%$. The content of PDA coating on the sand was calculated to be $3.3 \mathrm{wt} \%$, and the content of $\mathrm{Cu}$ ions on the PDA coated sand was $0.85 \mathrm{wt} \%$ (See supporting information). The specific surface areas of sand, PDA@sand, and Cu-PDA@sand were quantified by BET to be $1.3 \mathrm{~m}^{2} \mathrm{~g}^{-1}, 0.16 \mathrm{~m}^{2} \mathrm{~g}^{-1}$, and $0.04 \mathrm{~m}^{2} \mathrm{~g}^{-1}$. Their pore volumes were quantified to be $0.005 \mathrm{~cm}^{3}$ $\mathrm{g}^{-1}, 0.0004 \mathrm{~cm}^{3} \mathrm{~g}^{-1}$, and $0.0003 \mathrm{~cm}^{3} \mathrm{~g}^{-1}$, respectively. All these results from $\mathrm{N}_{2}$ adsorption reflect the pore-less structure and weak adsorbability of these sand-based catalysts towards dyes.

Furthermore, the valence state of $\mathrm{Cu}$ ions on the sand was further characterized by XPS. The characteristic shakeup satellite peaks (943-963 eV) confirmed the presence of $\mathrm{Cu}$ (II) (Fig. 1b) (Fan et al., 2009), and the peaks from $925 \mathrm{eV}$ to $938 \mathrm{eV}$ were assigned to $\mathrm{Cu} 2 \mathrm{p}_{3 / 2}$ (Fig. 1c). The lower peak at $934.2 \mathrm{eV}$ was attributed to $\mathrm{Cu}$ (II) from the prepared Cu-PDA@sand, and the

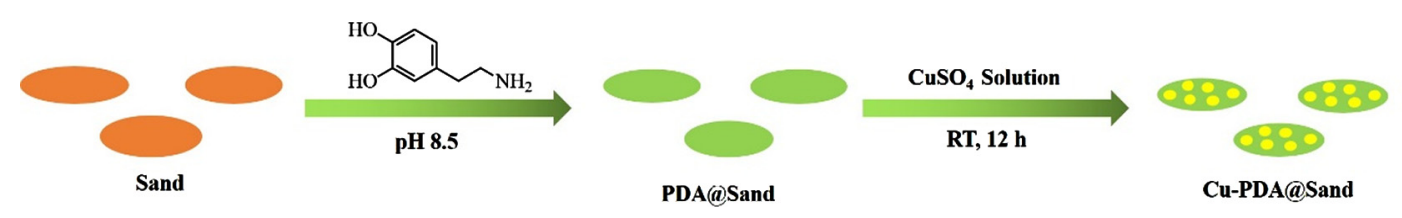

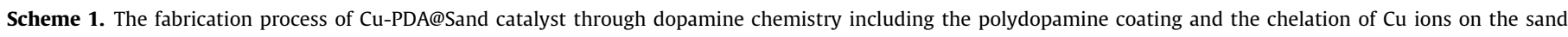
support. 

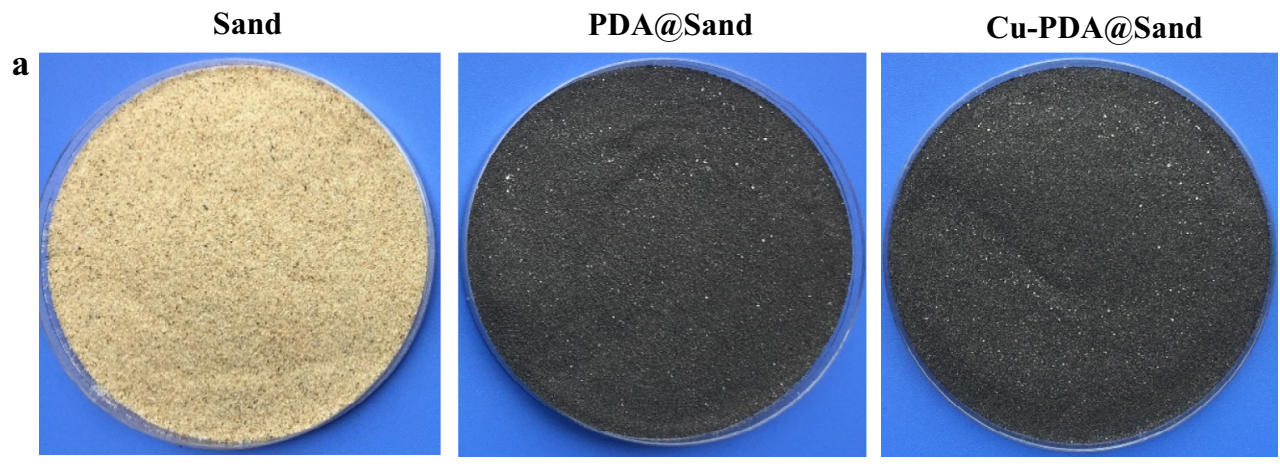

b

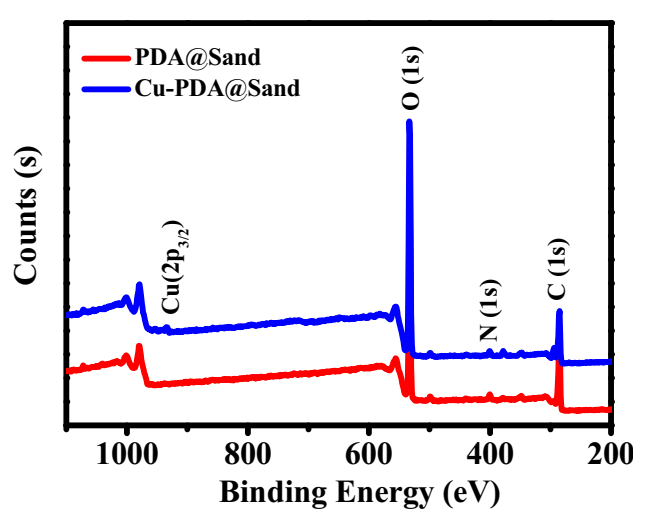

\begin{tabular}{lll}
\hline $\begin{array}{l}\text { Element } \\
(\%)\end{array}$ & PDA@Sand & $\begin{array}{l}\text { Cu-PDA } \\
\text { @Sand }\end{array}$ \\
\hline $\mathrm{Cu}$ & --- & 0.76 \\
$\mathrm{C}$ & 33.62 & 24.81 \\
$\mathrm{O}$ & 41.78 & 46.65 \\
$\mathrm{~N}$ & 2.46 & 1.78 \\
$\mathrm{Si}$ & 16.48 & 19.67 \\
$\mathrm{Al}$ & 4.92 & 4.84 \\
$\mathrm{~K}$ & 0.22 & 1.24 \\
$\mathrm{Na}$ & 0.52 & 0.53 \\
\hline
\end{tabular}

c

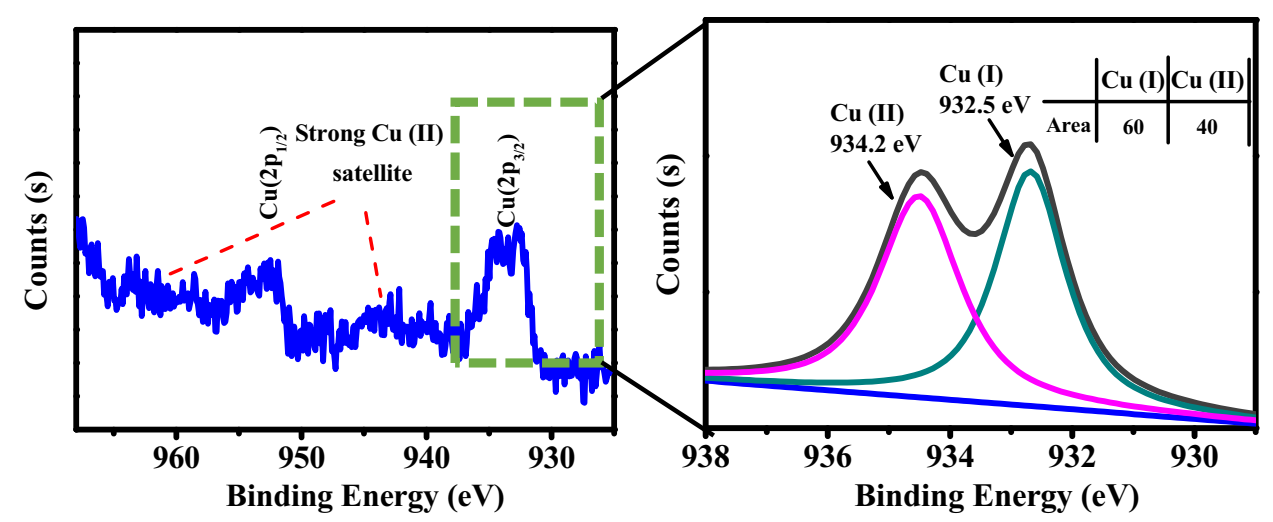

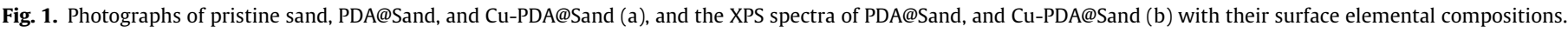
The enlarged spectra from $925 \mathrm{eV}$ to $970 \mathrm{eV}$ and the peak fitting of the $\mathrm{Cu} 2 \mathrm{p}_{3 / 2}$ spectrum of the Cu-PDA@Sand (c).

higher peak at $932.5 \mathrm{eV}$ indicated the presence of $\mathrm{Cu}(0)$ or $\mathrm{Cu}(\mathrm{I})$ in the Cu-PDA@sand. To further distinguish Cu (0) and Cu (I) species, $\mathrm{X}$-ray-induced auger electron spectroscopy was used and then confirmed the peak at $574 \mathrm{eV}$ from the characteristic $\mathrm{Cu}$ (I) peak (Fig. 2a). In addition, no characteristic peak of $\mathrm{Cu}(0)$ could be detected from the Cu-PDA@sand sample, indicating that $\mathrm{Cu}$ (II) was not reduced to $\mathrm{Cu}(0)$ through polydopamine. Therefore, it can conclude that the copper ions chelated on the PDA-coated sand is the mixture of $\mathrm{Cu}(\mathrm{I})$ and $\mathrm{Cu}$ (II) with $3: 2 \mathrm{M}$ fraction of $\mathrm{Cu}$ (I) over $\mathrm{Cu}$ (II) (Fig. 1c). Moreover, the content of $\mathrm{Cu}$ was determined to be $0.76 \mathrm{wt} \%$, which is almost the same with the results obtained from TGA (Fig. S5) and lower than $1.40 \%$ of Cu chelated on other substrates through dopamine chemistry in our previous work (Liu et al., 2016), probably due to the less surface area of sand for less PDA coating to support $\mathrm{Cu}$. Besides, the content fraction of $\mathrm{Si}$ and O elements was high up to $46.65 \%$ and $19.67 \%$, respectively, while that of $\mathrm{Al}$ element decreased to $4.84 \%$ after immobilization of $\mathrm{Cu}$ ions, probably due to the uneven chemical composition of natural sea sand.

\subsection{Catalytic properties of Cu-PDA@Sand}

The catalytic properties of the prepared Cu-PDA@Sand were investigated using organic dye intermediate 4-NP as a model. The reduction process of 4 -NP was monitored by the change of absorbance at $400 \mathrm{~nm}$. With the reduction of $4-\mathrm{NP}$ progressing, the 
a

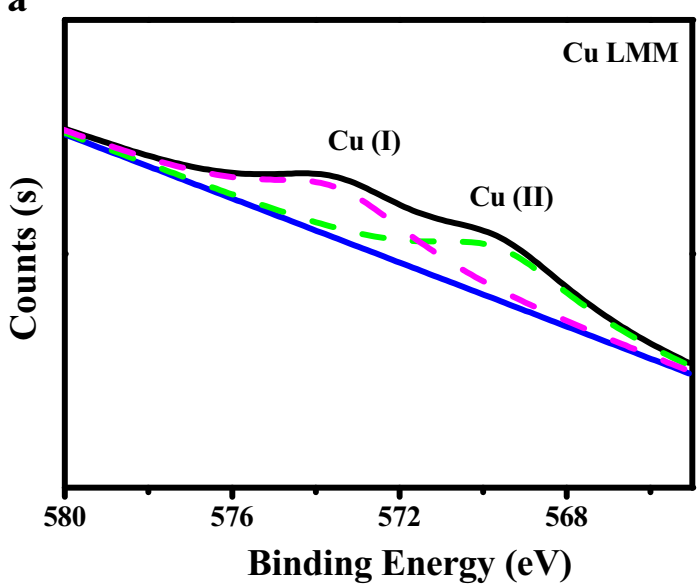

b

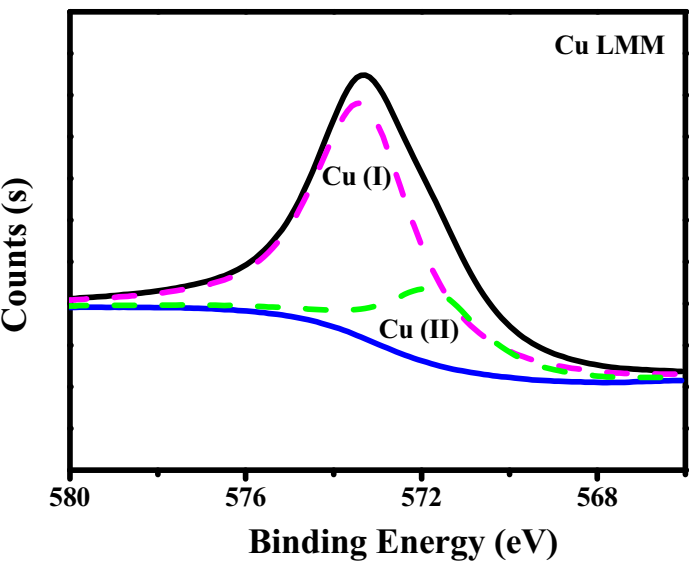

Fig. 2. Cu LMM XPS spectrum of $\mathrm{Cu}$ ions chelated onto the PDA@Sand (a) and the Cu-PDA@Sand after incubation with $\mathrm{NaBH}_{4}(\mathrm{~b})$.

green-yellow color of 4-NP solution completely disappeared as shown in Fig. 3a. The characteristic absorbance of 4-NP at $400 \mathrm{~nm}$ rapidly decreased to zero in $13 \mathrm{~min}$, indicating the complete degradation of 4-NP, but its absorbance at $300 \mathrm{~nm}$ gradually increased due to the formation of 4-aminophenol (4-AP) (Fig. 3b) (Zhang et al., 2014; Subair et al., 2016). To identify the valence state of $\mathrm{Cu}$ ions in the system after reaction with $\mathrm{NaBH}_{4}$, the Xray-induced Auger electron spectroscopy was also employed to detect no $\mathrm{Cu}(0)$ from Cu-PDA@Sand, but to observe the molar frac- tion of Cu (I) over Cu (II) on Cu-PDA@Sand increased from 3:2 to 4:1 (Fig. 2b). As literature reported that the catalytic rate of $\mathrm{Cu}$ (I) towards the reduction of 4-NP (12 min) (Huang et al., 2014) was much faster than that of $\mathrm{Cu}(0)$ (18 min) (Tang et al., 2014; Zhang et al., 2014). The $k_{a p p}\left(0.308 \mathrm{~min}^{-1}\right)$ in the Cu-PDA@Sand is much higher than the value of $\mathrm{Cu}(0)$ microspheres $\left(0.182 \mathrm{~min}^{-1}\right.$. Zhang et al., 2014) reported in the literature. The $k_{\text {app }}$ over Cu content was calculated as $202 \mathrm{~min}^{-1} / \mathrm{g} \mathrm{Cu}$, which is much higher than the reported results (36 $\mathrm{min}^{-1} / \mathrm{g} \mathrm{Cu}$, Zhang et al., 2014). The per- a

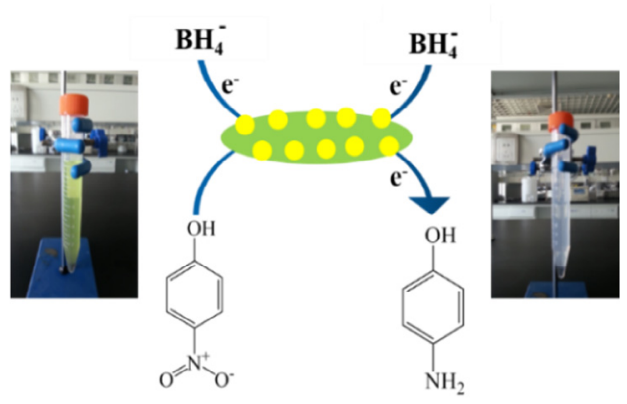

c

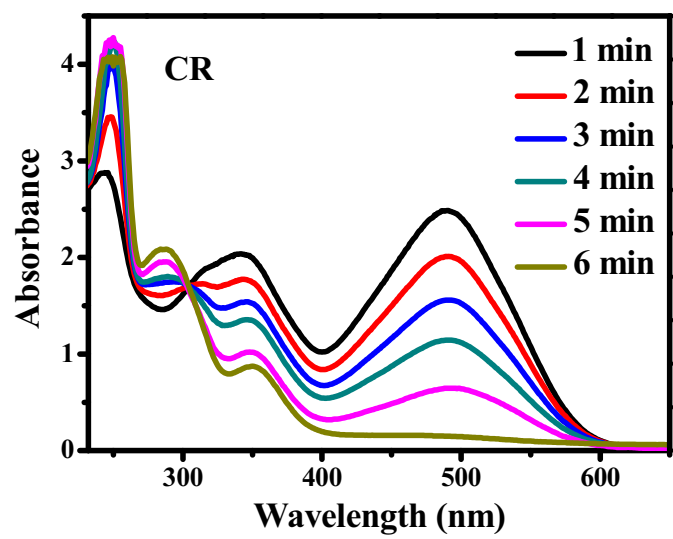

b

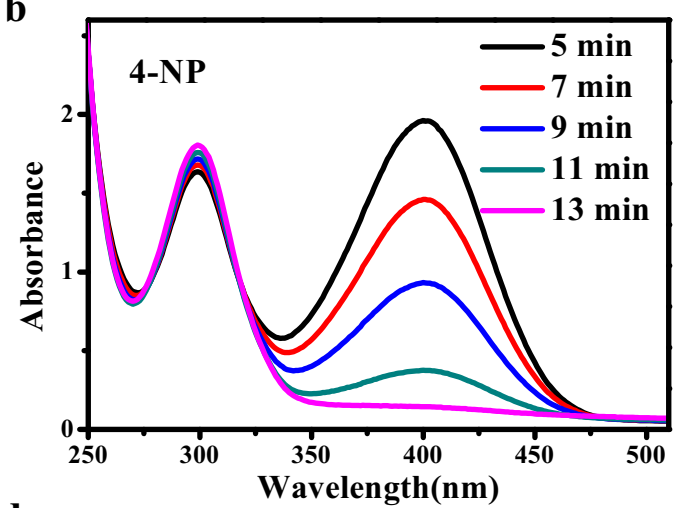

d

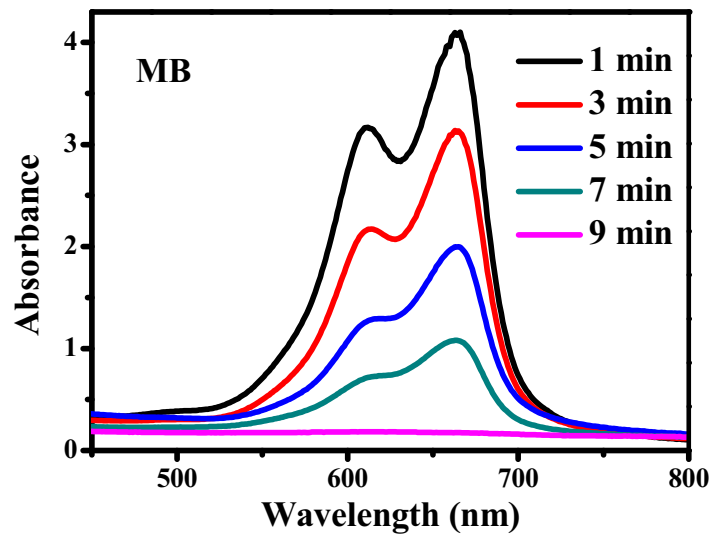

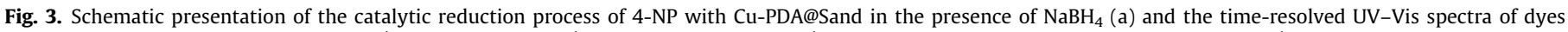
solutions containing (b) 4-NP $\left(100 \mathrm{mg} \mathrm{L}^{-1}\right)$, (c) CR $\left(100 \mathrm{mg} \mathrm{L}^{-1}\right)$, and (d) MB $\left(100 \mathrm{mg} \mathrm{L}^{-1}\right)$ with $\mathrm{NaBH}_{4}(0.02 \mathrm{M})$ and Cu-PDA@Sand (20 g L $\left.\mathrm{L}^{-1}\right)$. 
centage of Cu (I) in the Cu-PDA@Sand is 80 wt\%. We, therefore, propose that the high catalytic rate of Cu-PDA@Sand may be due to the high $\mathrm{Cu}$ (I) loading on the sand and a high efficiency of $\mathrm{Cu}(\mathrm{I})$.

To confirm the universality of the Cu-PDA@Sand as a catalyst for the reduction of organic dyes or intermediate, congo Red (CR) and methylene blue (MB) were also chosen as the model dyes. The catalytic reductions of $\mathrm{CR}$ and $\mathrm{MB}$ were also monitored by their solution optical density changes at their corresponding maximum absorbance wavelengths of CR $\left(\lambda_{510} \mathrm{~nm}\right)$ and $\mathrm{MB}\left(\lambda_{665} \mathrm{~nm}\right.$ and $\lambda_{614}$ $\mathrm{nm})$, respectively. Fig. $3 \mathrm{c}$ illustrates that the absorption peak of $\mathrm{CR}$ solution at $510 \mathrm{~nm}$ significantly decreased within $6 \mathrm{~min}$, indicating a very rapid reduction of CR in the presence of Cu-PDA@Sand and $\mathrm{NaBH}_{4}$. The UV-Vis spectrum of MB (Fig. 3d) exhibited two absorption peaks at $665 \mathrm{~nm}$ and $614 \mathrm{~nm}$, which are originated from the $\pi$ $\mathrm{p}^{*}$ transition and a shoulder peak, respectively. The color of the MB solution quickly faded within $9 \mathrm{~min}$ after reduced by $\mathrm{Cu}-$ PDA@Sand. No obvious new peak was observed in the spectrum, suggesting the complete reduction of MB to LMB (Zhang et al., 2014; Subair et al., 2016). All these results reveal that the prepared Cu-PDA@Sand exhibited high effective and universal catalytic activities, and reduced $C R$ and $M B$ in a much faster rate than 4NP under similar conditions.

The reduction kinetics of organic dyes were monitored and measured as the plot of dye concentration versus reaction time. Fig. 4a presents that the concentration of all dyes decreased exponentially with the reaction time and Fig. $4 \mathrm{~b}$ illustrates that $\ln \left(\mathrm{C}_{\mathrm{t}}\right)$ $\mathrm{C}_{\mathrm{o}}$ ) decreased linearly with the reaction time, which confirms that the catalytic reduction of organic dyes or intermediate follows the first order kinetics. The apparent reduction rates $k_{\text {app }}$ of three dyes or intermediate were calculated as the intercept of the plot of $\ln$ $\left(C_{t} / C_{o}\right)$ verse the reaction time following the reported Eq. (1). The $k_{\text {app }}$ of $\mathrm{CR}$ was $0.55 \mathrm{~min}^{-1}$, larger than $0.43 \mathrm{~min}^{-1}$ of $\mathrm{MB}$ and $0.34 \mathrm{~min}^{-1}$ of $4-\mathrm{NP}$, in the catalytic system of $20 \mathrm{~g} \mathrm{~L}^{-1} \mathrm{Cu}-$ PDA@Sand, $0.02 \mathrm{M} \mathrm{NaBH}_{4}$, and $100 \mathrm{mg} \mathrm{L}^{-1}$ dyes. In addition, the control experiment using PDA@Sand rather than Cu-PDA@Sand, both the absorbance of the 4-NP solution at $400 \mathrm{~nm}$ and the solution color did not change with time in the presence of $\mathrm{NaBH}_{4}$.

The catalytic reduction process of copper ions is an electrochemical mechanism where copper ions work as an electron relay from $\mathrm{NaBH}_{4}$ to organic dyes, and the concentrations of $\mathrm{Cu}$ PDA@Sand, dyes, and $\mathrm{NaBH}_{4}$ may greatly affect the reduction rate. The dependences of $k_{a p p}$ on the concentrations of Cu-PDA@Sand, dyes, and $\mathrm{NaBH}_{4}$ were investigated, and these results are shown in Fig. 5 and Fig. S6, using the 4-NP and CR as the models. Fig. 5a finds that the $k_{a p p}$ of 4-NP increased linearly as the concentration of Cu-PDA@Sand increased to $40 \mathrm{~g} \mathrm{~L}^{-1}$, and then almost unaltered with the further increased concentration of Cu-PDA@Sand. Our previous work has demonstrated that the unaltered $k_{\text {app }}$ was due to the lack of reductant $\mathrm{NaBH}_{4}$ (Wang et al., 2017). Moreover, it was also found that the $k_{\text {app }}$ decreased rapidly with the increasing 4-NP concentration and then reached to a plateau when 4-NP was more than $200 \mathrm{mg} \mathrm{L}^{-1}$ (Fig. 5b), and the $k_{\text {app }}$ increased obiviously with the $\mathrm{NaBH}_{4}$ concentration (Fig. 5c). Additionally, the catalytic reduction of $\mathrm{CR}$ also exhibited very similar phenomena as shown in Fig. S6. It can be concluded that the catalytic activity of $\mathrm{Cu}-$ PDA@Sand could be well optimized to simply tailor the concentration of Cu-PDA@Sand, dyes, and $\mathrm{NaBH}_{4}$. Generally, the concentration of the reductant $\mathrm{NaBH}_{4}$ was added significantly more than enough to allow the catalytic rate of dyes depend entirely on the concentration of catalysts. For example, $k_{\text {app }}$ of $1.28 \mathrm{~min}^{-1}$ is obtained through optimizing the condition with $20 \mathrm{~g} \mathrm{~L}^{-1} \mathrm{Cu}-$ PDA@Sand, $100 \mathrm{mg} \mathrm{L}^{-1} 4-\mathrm{NP}$, and $0.04 \mathrm{M} \mathrm{NaBH}_{4}$, which is much higher than those $\left(0.18 \mathrm{~min}^{-1}\right.$ (Zhang et al., 2014), $0.35 \mathrm{~min}^{-1}$ (Huang et al., 2014), and $0.50 \mathrm{~min}^{-1}$ (Huang et al., 2012)) reported elsewhere. The high catalytic rate of Cu-PDA@Sand is probably due to the high $\mathrm{Cu}(\mathrm{I})$ ions loading on the sand.

In addition, the dependence of $k_{a p p}$ on $\mathrm{pH}$ was investigated using MB as a model dye. Results are shown in Fig. S7. $k_{a p p}$ decreased from $0.75 \mathrm{~min}^{-1}$ to $0.31 \mathrm{~min}^{-1}$ as the increasing of $\mathrm{pH}$ value from $3,5,7$, to 9 , which may be attributed to the strong binding capabilities of polydopamine towards $\mathrm{Cu}$ ions at high $\mathrm{pH}$ conditions ((Liu et al., 2014).

\subsection{Long-term catalytic activity and recyclability of Cu-PDA@Sand}

The long-term catalytic activity and recyclability of the catalysts are key properties to determine their potentials in practical applications (Dong et al., 2014). The long-term stability and catalytic activity of Cu-PDA@Sand were investigated upon soaking it in water for 30 days. As shown in Fig. 6a, all the $k_{\text {app }}$ of $\mathrm{Cu}-$ PDA@Sand towards organic dyes or intermediate did not change much after 30 days soaking. The $k_{a p p}$ decreased slightly within $6 \%$ with no statistical difference for 4-NP, CR, and MB, respectively, which confirms that the catalytic performances of Cu-PDA@Sand were efficient and stable in the long-term.

Cu-PDA@Sand was found to be easily collected and reused for the next cycle just by self-sedimentation in aqueous solution because of the high density of sand, which provides a very simple and convenient process to recover it (Fig. 3a). Successive 20 cycles of Cu-PDA@Sand were tested to measure its catalytic properties for a

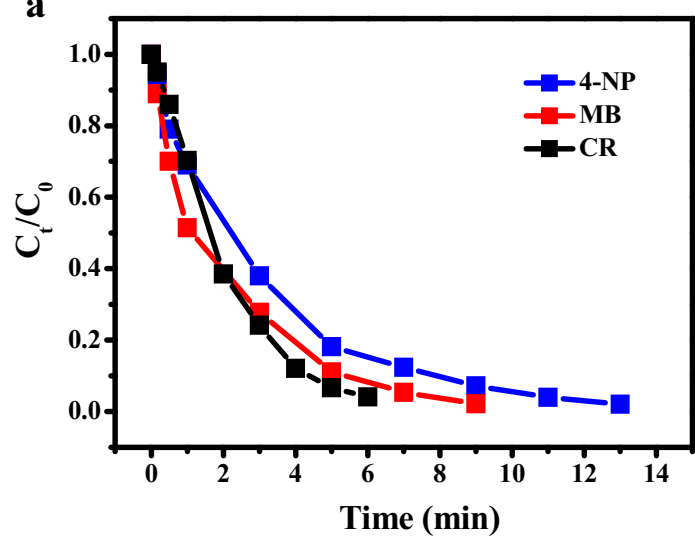

b

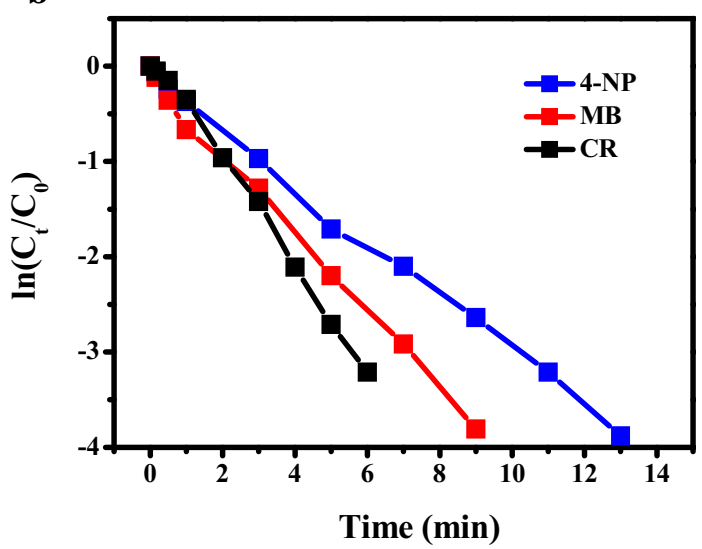

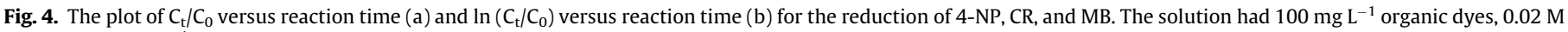
$\mathrm{NaBH}_{4}$, and $20 \mathrm{~g} \mathrm{~L}^{-1} \mathrm{Cu}-\mathrm{PDA} @$ Sand. 

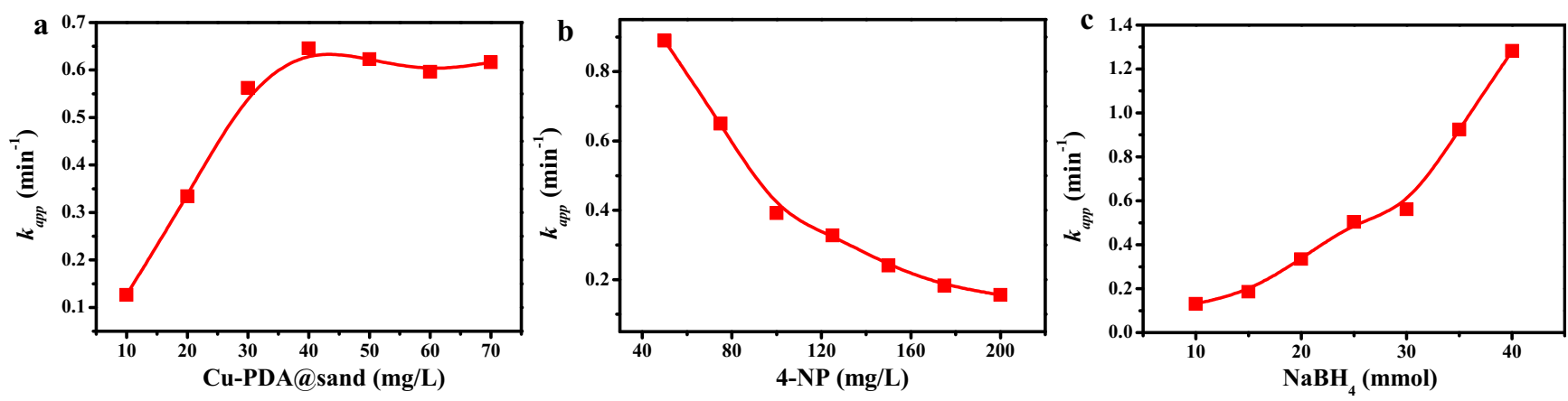

Fig. 5. The apparent reduction rate constant $\left(k_{a p p}\right)$ of 4-NP as a function of the concentration of Cu-PDA@Sand (a), 4-NP (b), and NaBH 4 (c), respectively.
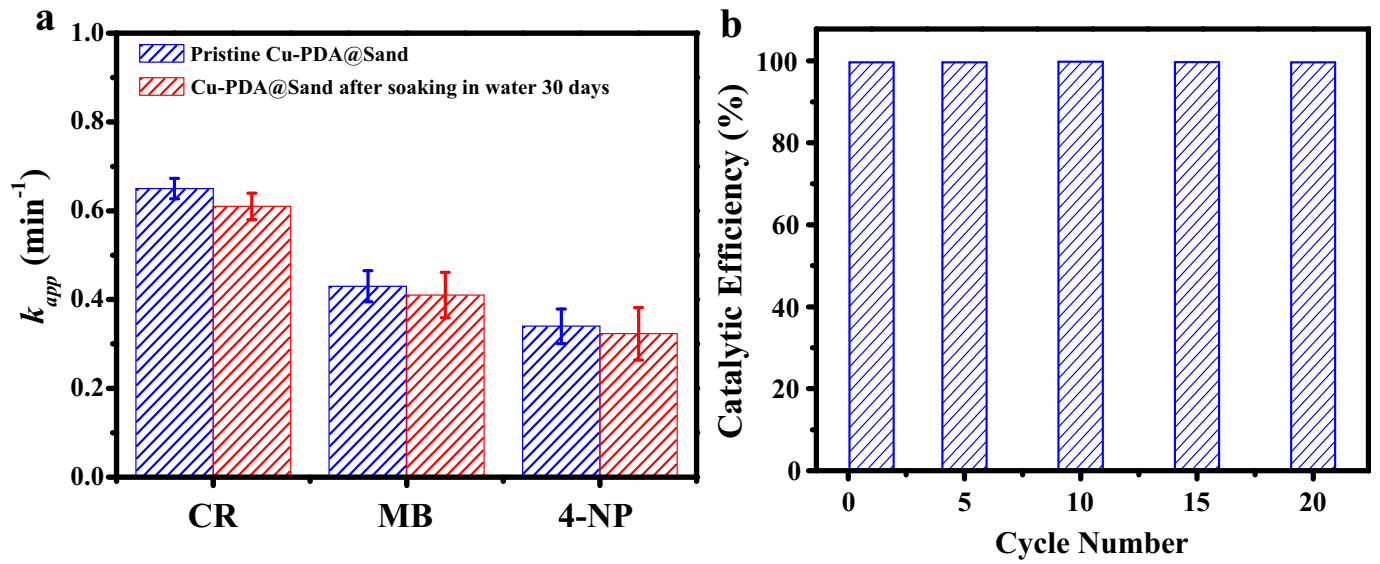

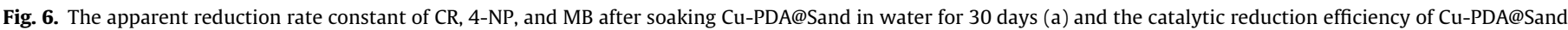
towards 4-NP during the tested successive 20 cycles (b). The catalytic conditions: $20 \mathrm{~g} / \mathrm{L}$ Cu-PDA@Sand, 0.02 M NaBH 4 , and 100 mg/L organic dyes.

recyclable and sustainable use, 4-NP was selected as a model dye. Fig. 6b shows that Cu-PDA@Sand kept highly effective and stable catalytic efficiency of $100 \%$ towards 4 -NP during the tested 20 cycles and could completely fade the 4-NP solution in each cycle, which was much better than the reported unstable $\mathrm{CuFe}_{2} \mathrm{O}_{4}$ catalyst during the catalytic process without recyclability (Feng et al., 2013). Overall, the easy-prepared low-cost Cu-PDA@Sand catalyst showed not only highly effective and sustainable catalytic activities but also recyclable and long-term stable properties, exhibiting a great potential of practical applications for color removal of the dye-containing wastewater.

\section{Conclusion and implication}

Our work has demonstrated that $\mathrm{Cu}$ ions could be successfully chelated onto the sand (Cu-PDA@Sand) through the facile and universal dopamine chemistry, followed by the incubation with a cupric salt solution. The copper ions chelated on the PDA-coated sand are the mixture of $\mathrm{Cu}$ (I) and $\mathrm{Cu}$ (II) $\mathrm{w}$ and the molar fraction of $\mathrm{Cu}$ (I) over Cu (II) was increased from the initial 3:2 to 4:1 but with no $\mathrm{Cu}(0)$ found during the catalytic reduction process in the presence of $\mathrm{NaBH}_{4}$. 4-Nitrophenol, methylene blue, and congo red were selected as the model dyes to confirm the universality of the $\mathrm{Cu}$ PDA@Sand as a catalyst towards their decoloration. The catalytic reduction of these organic dyes or intermediate follows the first order kinetics. The apparent reduction rate $k_{a p p}$ of $\mathrm{CR}$ was $0.65 \mathrm{~min}^{-1}$, larger than $0.43 \mathrm{~min}^{-1}$ of $\mathrm{MB}$ and $0.34 \mathrm{~min}^{-1}$ of $4-\mathrm{NP}$, in the catalytic system of Cu-PDA@Sand $\left(20 \mathrm{~g} \mathrm{~L}^{-1}\right), \mathrm{NaBH}_{4}$ $(0.02 \mathrm{M})$, and dyes with initial dye concentration $100 \mathrm{mg} \mathrm{L}^{-1}$. Fur- thermore, $k_{\text {app }}$ was highly dependent on the concentrations of $\mathrm{Cu}$ PDA@Sand, dyes, and $\mathrm{NaBH}_{4}$ when using the 4-NP and CR as the model dyes. The $k_{\text {app }}$ of 4-NP increased with the concentration of Cu-PDA@Sand and $\mathrm{NaBH}_{4}$, but decreased with the concentration of dyes, and may reach to a plateau when either Cu-PDA@Sand or $\mathrm{NaBH}_{4}$ was significantly less than the required amount to reduce dyes. The optimized $k_{a p p}$ of 4-NP could reach to $1.28 \mathrm{~min}^{-1}$ (20 g $\mathrm{L}^{-1} \mathrm{Cu}$-PDA@Sand, $100 \mathrm{mg} \mathrm{L}^{-1}$ 4-NP, and $\left.0.04 \mathrm{M} \mathrm{NaBH}_{4}\right)$, which is much higher than the reported results from literature. The high catalytic rate of Cu-PDA@Sand is probably due to the high $\mathrm{Cu}$ (I) ions loading on the sand.

Meanwhile, the recyclability of Cu-PDA@Sand was tested and the reduction of 4-NP was measured when Cu-PDA@Sand was used for $5,10,15$, and 20 cycles, the results suggest that the reduction efficiency of Cu-PDA@Sand maintained 100\% during the tested successive 20 cycles. Additionally, the long-term stability and catalytic activities of Cu-PDA@Sand were also evaluated after soaking CuPDA@Sand in water for 30 days, the results confirm that the catalyst did not show a statistical decrease towards the reduction of 4-NP, MB, and CR. All these results illustrate that Cu-PDA@Sand can be easily prepared with low cost and simply recovered through self-sedimentation, and reused for long-term stable and efficient catalytic performances, which presents a great potential of $\mathrm{Cu}-$ PDA@Sand in the practical applications for color removal of the dye-containing wastewater.

As literature study confirmed the versatile and universal PDA coatings could be deposited on the surface of diverse substrates through dopamine chemistry including organic, inorganic, and other materials, the copper ions chelation is mainly through the PDA coating, and thus copper ions can be chelated on diverse sub- 
strates through dopamine chemistry and then used as catalysts, biocides, and other functional materials. Our facile and universal approach of copper ions chelation may open a new avenue to fabricate the low-cost copper-based materials on a large scale for practical applications.

\section{Acknowledgments}

The authors gratefully acknowledge the funding support from National Natural Science Foundation of China (No. 21476249), Chang-jiang Scholars and Innovative Research Team in the University of Ministry of Education, China (No. IRT-17R80), Program for Innovative Research Team in University of Tianjin (No. TD135044), the Science and Technology Plans of Tianjin (No.17PTSYJC00060), and Key Science and Technology Program of Yantai City (No. 2018ZHGY075).

\section{Declaration of interest statement}

This manuscript is solely the work of the authors and the authors declare no competing financial interest.

\section{Appendix A. Supplementary material}

Supplementary data to this article can be found online at https://doi.org/10.1016/j.ces.2019.04.009.

\section{References}

Ali, I., 2012. New Generation Adsorbents for Water Treatment. Chemical Reviews 112, 5073-5091.

Ayati, A., Shahrak, M.N., Tanhaei, B., Sillanpää, M., 2016. Emerging adsorptive removal of azo dye by metal-organic frameworks. Chemosphere 160, 30-44.

Bastús, N.G., Merkoçi, F., Piella, J., Puntes, V., 2014. Synthesis of highly monodisperse citrate-stabilized silver nanoparticles of up to $200 \mathrm{~nm}$ : kinetic control and catalytic properties. Chem. Mater. 26, 2836-2846.

Bradu, C., Frunza, L., Mihalche, N., Avramescu, S.-M., Neaţă, M., Udrea, I., 2010 Removal of Reactive Black 5 azo dye from aqueous solutions by catalytic oxidation using $\mathrm{CuO} / \mathrm{Al}_{2} \mathrm{O}_{3}$ and $\mathrm{NiO} / \mathrm{Al}_{2} \mathrm{O}_{3}$. Appl. Catal. B 96, 548-556.

Chaplin, B.P., Reinhard, M., Schneider, W.F., Schüth, C., Shapley, J.R., Strathmann, T.J. Werth, C.J., 2012. Critical review of Pd-based catalytic treatment of priority contaminants in water. Environ. Sci. Tech. 46, 3655-3670.

de Luna, L.A.V., da Silva, T.H.G., Nogueira, R.F.P., Kummrow, F., Umbuzeiro, G.A., 2014. Aquatic toxicity of dyes before and after photo-Fenton treatment. J. Hazard. Mater. 276, 332-338.

Dong, Z., Le, X., Li, X., Zhang, W., Dong, C., Ma, J., 2014. Silver nanoparticles immobilized on fibrous nano-silica as highly efficient and recyclable heterogeneous catalyst for reduction of 4-nitrophenol and 2-nitroaniline. Appl. Catal. B 158-159, 129-135.

Eggen, R.I.L., Hollender, J., Joss, A., Schärer, M., Stamm, C., 2014. Reducing the discharge of micropollutants in the aquatic environment: the benefits of upgrading wastewater treatment plants. Environ. Sci. Tech. 48, 7683-7689.

Fan, J., Dai, Y., Li, Y., Zheng, N., Guo, J., Yan, X., Stucky, G.D., 2009. Low-temperature highly selective, gas-phase oxidation of benzyl alcohol over mesoporous K-Cu$\mathrm{TiO}_{2}$ with stable copper (I) oxidation state. J. Am. Chem. Soc. 131, 15568-15569.

Feng, J., Su, L., Ma, Y., Ren, C., Guo, Q., Chen, X., 2013. $\mathrm{CuFe}_{2} \mathrm{O}_{4}$ magnetic nanoparticles: a simple and efficient catalyst for the reduction of nitrophenol. Chem. Eng. J. 221, 16-24.

Gao, S., Zhang, Z., Liu, K., Dong, B., 2016. Direct evidence of plasmonic enhancement on catalytic reduction of 4-nitrophenol over silver nanoparticles supported on flexible fibrous networks. Appl. Catal B 188, 245-252.

Ghosh, B.K., Hazra, S., Naik, B., Ghosh, N.N., 2015. Preparation of Cu nanoparticle loaded SBA-15 and their excellent catalytic activity in reduction of variety of dyes. Powder Technol. 269, 371-378.

Hassan, M.M., Carr, C.M., 2018. A critical review on recent advancements of the removal of reactive dyes from dyehouse effluent by ion-exchange adsorbents. Chemosphere 209, 201-219.

Hu, H.W., Xin, J.H., Hu, H., 2013. Highly efficient graphene-based ternary composite catalyst with polydopamine layer and copper nanoparticles. ChemPlusChem 78 1483-1490.

Huang, C., Ye, W., Liu, Q., Qiu, X., 2014. Dispersed $\mathrm{Cu}_{2} \mathrm{O}$ octahedrons on h-BN nanosheets for p-nitrophenol reduction. ACS Appl. Mater. Interf. 6, 1446914476.

Huang, X., Li, Y., Zhou, H., Zhong, X., Duan, X., Huang, Y., 2012. Simplifying the creation of dumbbell-like $\mathrm{Cu}-\mathrm{Ag}$ nanostructures and their enhanced catalytic activity. Chem-Eur. J. 18, 9505-9510.
Kurt, E., Koseoglu-Imer, D.Y., Dizge, N., Chellam, S., Koyuncu, I., 2012. Pilot-scale evaluation of nanofiltration and reverse osmosis for process reuse of segregated textile dyewash wastewater. Desalination 302, 24-32.

Laszlo, J.A., 2000. Regeneration of azo-dye-saturated cellulosic anion exchange resin by Burkholderia cepacia anaerobic dye reduction. Environ. Sci. Tech. 34, 167172.

Li, J., Liu, C.Y., Liu, Y., 2012. Au/graphene hydrogel: synthesis, characterization and its use for catalytic reduction of 4-nitrophenol. J. Mater. Chem. 22, 8426-8430.

Lipatova, I.M., Makarova, L.I., Yusova, A.A., 2018. Adsorption removal of anionic dyes from aqueous solutions by chitosan nanoparticles deposited on the fibrous carrier. Chemosphere 212, 1155-1162.

Liu, Z., Hu, Y. Liu, C., Zhou, Z., 2016. Surface-independent one-pot chelation of copper ions onto filtration membranes to provide antibacterial properties. Chem. Commun. 52, 12245-12248.

Luo, J., Zhang, N., Liu, R., Liu, X., 2014. In situ green synthesis of Au nanoparticles onto polydopamine-functionalized graphene for catalytic reduction of nitrophenol. RSC Adv. 4, 64816-64824.

Liu, Y., Ai, K., Lu, L., 2014. Polydopamine and its derivative materials: synthesis and promising applications in energy, environmental, and biomedical fields. Chem. Rev. 114, 5057-5115.

Okesola, B.O., Smith, D.K., 2016. Applying low-molecular weight supramolecular gelators in an environmental setting-self-assembled gels as smart materials for pollutant removal. Chem. Soc. Rev. 45, 4226-4251.

Ong, Y.K., Li, F.Y., Sun, S.P., Zhao, B.W., Liang, C.Z., Chung, T.S., 2014. Nanofiltration hollow fiber membranes for textile wastewater treatment: lab-scale and pilotscale studies. Chem. Eng. Sci. 114, 51-57.

Panáček, A., Prucek, R., Hrbáč, J., Nevečná, T.j., Šteffková, J., Zbořil, R., Kvítek, L., 2014. Polyacrylate-assisted size control of silver nanoparticles and their catalytic activity. Chem. Mater. 26, 1332-1339.

Subair, R., Tripathi, B.P., Formanek, P., Simon, F., Uhlmann, P., Stamm, M., 2016. Polydopamine modified membranes with in situ synthesized gold nanoparticles for catalytic and environmental applications. Chem. Eng. J. 295, 358-369.

Subramanian, V. Wolf, E.E. Kamat, P.V., 2004. Catalysis with $\mathrm{TiO}_{2} /$ gold nanocomposites. Effect of metal particle size on the Fermi level equilibration. J. Am. Chem. Soc. 126, 4943-4950.

Šuláková, R., Hrdina, R., Soares, G.M.B., 2007. Oxidation of azo textile soluble dyes with hydrogen peroxide in the presence of $\mathrm{Cu}$ (II)-chitosan heterogeneous catalysts. Dyes Pigments 73, 19-24.

Sun, H., Liu, S., Liu, S., Wang, S., 2014. A comparative study of reduced graphene oxide modified $\mathrm{TiO}_{2}, \mathrm{ZnO}$ and $\mathrm{Ta}_{2} \mathrm{O}_{5}$ in visible light photocatalytic/ photochemical oxidation of methylene blue. Appl. Catal. B 146, 162-168.

Sun, Y., Xu, L., Yin, Z., Song, X., 2013. Synthesis of copper submicro/nanoplates with high stability and their recyclable superior catalytic activity towards 4nitrophenol reduction. J. Mater. Chem. A 1, 12361-12370.

Szpyrkowicz, L., Juzzolino, C., Kaul, S.N., 2001. A comparative study on oxidation of disperse dyes by electrochemical process, ozone, hypochlorite and Fenton reagent. Water Res. 35, 2129-2136.

Tang, J., Shi, Z., Berry, R.M., Tam, K.C., 2015. Mussel-inspired green metallization of silver nanoparticles on cellulose nanocrystals and their enhanced catalytic reduction of 4-nitrophenol in the presence of $\beta$-cyclodextrin. Ind. Eng. Chem. Res. 54, 3299-3308.

Tang, M., Zhang, S., Li, X., Pang, X., Qiu, H., 2014. Fabrication of magnetically recyclable $\mathrm{Fe}_{3} \mathrm{O}_{4} @ \mathrm{Cu}$ nanocomposites with high catalytic performance for the reduction of organic dyes and 4-nitrophenol. Mater. Chem. Phys. 148, 639-647.

Verma, A.K., Dash, R.R., Bhunia, P., 2012. A review on chemical coagulation/ flocculation technologies for removal of colour from textile wastewaters. J. Environ. Manag. 93, 154-168.

Wang, N., Hu, Y., Zhang, Z., 2017. Sustainable catalytic properties of silver nanoparticles supported montmorillonite for highly efficient recyclable reduction of methylene blue. Appl. Clay Sci. 150, 47-55.

Wang, X., Jiang, C., Hou, B., Wang, Y., Hao, C., Wu, J., 2018. Carbon composite ligninbased adsorbents for the adsorption of dyes. Chemosphere 206, 587-596.

Xie, Y., Yan, B., Xu, H., Chen, J., Liu, Q., Deng, Y., Zeng, H., 2014. Highly regenerable mussel-inspired $\mathrm{Fe}_{3} \mathrm{O}_{4} @$ polydopamine-Ag core-shell microspheres as catalyst and adsorbent for methylene blue removal. ACS Appl. Mater. Interf. 6, 88458852 .

Yao, T., Cui, T., Wang, H., Xu, L., Cui, F., Wu, J., 2014. A simple way to prepare Au@polypyrrole $/ \mathrm{Fe}_{3} \mathrm{O}_{4}$ hollow capsules with high stability and their application in catalytic reduction of methylene blue dye. Nanoscale 6, 7666-7674.

Zeng, T., Niu, H.Y., Ma, Y.R., Li, W.H., Cai, Y.Q., 2013. In situ growth of gold nanoparticles onto polydopamine-encapsulated magnetic microspheres for catalytic reduction of nitrobenzene. Appl. Catal. B 134, 26-33.

Zhang, C., Li, Y., Li, Y., Zhang, W., Wang, X., He, X., Yu, M., 2019. Synthesis and Zn(II) modification of hierarchical porous carbon materials from petroleum pitch for effective adsorption of organic dyes. Chemosphere 216, 379-386.

Zhang, P., Sui, Y., Xiao, G., Wang, Y., Wang, C., Liu, B., Zou, G., Zou, B., 2013. Facile fabrication of faceted copper nanocrystals with high catalytic activity for pnitrophenol reduction. J. Mater. Chem. A 1, 1632-1638.

Zhang, X., Huang, Q., Liu, M., Tian, J., Zeng, G., Li, Z., Wang, K., Zhang, Q., Wan, Q., Deng, F., Wei, Y., 2015. Preparation of amine functionalized carbon nanotubes via a bioinspired strategy and their application in $\mathrm{Cu}^{2+}$ removal. Appl. Surf. Sci. $343,19-27$.

Zhang, X., Huang, Q., Deng, F., Huang, H., Wan, Q., Liu, M., Wei, Y., 2017. Musselinspired fabrication of functional materials and their environmental applications: Progress and prospects. Appl. Mater. Today 7, 222-238. 
Zhang, Y., Liu, S., Lu, W., Wang, L., Tian, J., Sun, X., 2011. In situ green synthesis of Au nanostructures on graphene oxide and their application for catalytic reduction of 4-nitrophenol. Catal. Sci. Technol. 1, 1142-1144.

Zhang, Y., Zhu, P., Chen, L., Li, G., Zhou, F., Lu, D.D., Sun, R., Zhou, F., Wong, C.P., 2014 Hierarchical architectures of monodisperse porous $\mathrm{Cu}$ microspheres: synthesis, growth mechanism, high-efficiency and recyclable catalytic performance. J. Mater. Chem. A 2, 11966-11973.
Zheng, Y., Wang, A., 2012. Ag nanoparticle-entrapped hydrogel as promising material for catalytic reduction of organic dyes. J. Mater. Chem. A 22, $16552-$ 16559.

Zhu, M., Wang, C., Meng, D., Diao, G., 2013. In situ synthesis of silver nanostructures on magnetic $\mathrm{Fe}_{3} \mathrm{O}_{4} @ \mathrm{C}$ core-shell nanocomposites and their application in catalytic reduction reactions. J. Mater. Chem. A 1, 2118-2125. 